\title{
Evaluation of Ventilator-Associated Tracheitis in Children with Pre-Existing Tracheostomies: Organisms and Empiric Coverage
}

\author{
Victoria Urban ${ }^{1}$, Christopher Campbell ${ }^{2}$, Jennifer Waller ${ }^{1}$, and Kelley Norris ${ }^{2}$ \\ ${ }^{1}$ Augusta University Medical Center \\ ${ }^{2}$ Children's Hospital of Georgia
}

June 23, 2021

\begin{abstract}
Evaluation of Ventilator-Associated Tracheitis in Children with Pre-Existing Tracheostomies: Organisms and Empiric Coverage Objectives: This study aims to describe the outcomes, microbiome, and empiric antimicrobial coverage of ventilator associated tracheitis (VAT) in pediatric patients with pre-existing tracheostomies. Patients and Methods: This is a retrospective chart review of pediatric patients with pre-existing tracheostomies admitted to the Children's Hospital of Georgia for initial inpatient treatment for VAT between January 1, 2007 and February 21, 2021. Patients were evaluated for incidence of ventilator associated pneumonia (VAP), tracheostomy culture results, and antibiotic choice. Results: Thirty-nine patients were included. Across all patients evaluated with pre-existing tracheostomies, 60\% developed VAT. There was no difference in the development in VAP between shorter and longer treatment durations ( 0 vs $1, \mathrm{p}=1$ ). Patients who developed Pseudomonas aeruginosa VAT were likely to have a previous culture of P. aeruginosa $(\mathrm{p}=0.003)$, have a tracheostomy for longer $(\mathrm{p}=0.011)$, and be older than 1 year of age $(\mathrm{p}=0.0002)$. MRSA VAT was associated with a previous culture growing MRSA $(\mathrm{p}=0.0042)$. Conclusions: VAT incidence was higher than what was shown in pediatric patients without pre-existing tracheostomies but VAP incidence was lower and there was no difference between treatment groups. VAT should be treated based on previous cultures and Pseudomonas aeruginosa should be considered as a causative organism.
\end{abstract}

Evaluation of Ventilator-Associated Tracheitis in Children with Pre-Existing Tracheostomies: Organisms and Empiric Coverage

Victoria Urban, PharmD ${ }^{1}$, Christopher Campbell, PharmD, BCPPS ${ }^{2}$, Jennifer L. Waller, PhD,${ }^{3}$ Kelley Norris, PharmD, BCPS, BCPPS ${ }^{2}$

1. Augusta University Medical Center, The University of Georgia College of Pharmacy

2. Children's Hospital of Georgia

3. Augusta University, Medical College of Georgia, Department of Population Health Sciences, Division of Biostatistics and Data Science

Presented briefly at Southeastern Residency Conference (SERC).

Keywords:

- Tracheitis

- Tracheostomy

- Pseudomonas aeruginosa

- Methicillin-resistance staphylococcus aureus

Send requests to: Victoria Urban

$112015^{\text {th }}$ Street Augusta GA 30901 
Phone: 302-507-5069

VictoriaMurban@gmail.com

Abbreviated Title: Pediatric VAT and Tracheostomies

Abstract :

Evaluation of Ventilator-Associated Tracheitis in Children with Pre-Existing Tracheostomies: Organisms and Empiric Coverage

Objectives: This study aims to describe the outcomes, microbiome, and empiric antimicrobial coverage of ventilator associated tracheitis (VAT) in pediatric patients with pre-existing tracheostomies.

Patients and Methods: This is a retrospective chart review of pediatric patients with pre-existing tracheostomies admitted to the Children's Hospital of Georgia for initial inpatient treatment for VAT between January 1, 2007 and February 21, 2021. Patients were evaluated for incidence of ventilator associated pneumonia (VAP), tracheostomy culture results, and antibiotic choice.

Results: Thirty-nine patients were included. Across all patients evaluated with pre-existing tracheostomies, $60 \%$ developed VAT. There was no difference in the development in VAP between shorter and longer treatment durations ( 0 vs $1, \mathrm{p}=1.00)$. Patients who developedPseudomonas aeruginosa VAT were likely to have a previous culture of $P$. aeruginosa $(\mathrm{p}=0.0002)$, have a tracheostomy for longer $(\mathrm{p}=0.012)$, and be older than 1 year of age $(\mathrm{p}=0.0002)$. MRSA VAT was associated with a previous culture growing MRSA $(\mathrm{p}=$ $0.0042)$.

Conclusions: VAT incidence was higher than what was shown in pediatric patients without pre-existing tracheostomies but VAP incidence was lower and there was no difference between treatment groups. VAT should be treated based on previous cultures and Pseudomonas aeruginosa should be considered as a causative organism.

\section{Introduction}

Ventilator associated tracheitis (VAT) is common in intubated patients, occurring in approximately $3.4 \%$ of pediatric intensive care unit patients. ${ }^{1}$ A patient with VAT may present with fever, leukocytosis or leukopenia, increased production of purulent sputum, and positive endotracheal aspirate culture. ${ }^{2}$ VAT is associated with an increased risk of ventilator associated pneumonia (VAP) but is differentiated from VAP by the absence of radiologic evidence of pneumonia. ${ }^{2}$ Ventilator-associated pneumonia is a pneumonia occurring at least 48 hours after intubation and mechanical ventilation. ${ }^{3}$

In adult patients, the duration of VAT treatment is not well defined. Many adult VAT studies have assessed whether treatment of VAT is necessary, rather than the optimal duration of therapy. ${ }^{4,5}$ A prospective observational multicenter study assessed adult patients experiencing a first episode of VAT in intensive care units (ICUs). There was a significant difference in percentage of patients receiving appropriate antimicrobial treatment who did not develop VAP (11\%) compared to those who did develop VAP $(53 \%) .{ }^{5}$ However, this study was an observational study and excluded patients with tracheostomies. ${ }^{5}$

A prospective, multi-center randomized controlled trial assessed the benefit of treating VAT with intravenous antibiotics for 8 days compared to not treating at all. ${ }^{6}$ VAP development from the same organism causing VAT was significantly lower in the antibiotic group compared to the no antibiotic group ( $0 \%$ ( 0 of 3 ) vs $82 \%$ (14 of 17), $\mathrm{p}=0.018$ ). Rates of VAP, in general, were lower in the antibiotic group (13\% (3 of 22 ) vs $47 \%$ (17 of 36$), \mathrm{p}=0.011$ ). ICU mortality was also significantly lower in the antibiotic treatment group (18\% (4 of 22 ) vs $47 \%$ (17 of 36 ), $\mathrm{p}=0.047) .{ }^{6}$ In this study, patients with pre-existing tracheostomies were also excluded.

Similarly, in pediatric patients, the duration of VAT treatment is not well defined. A retrospective cohort study showed that patients treated for VAT greater than 7 days did not have a lower incidence of VAP compared to those treated for 7 days or less. ${ }^{7}$ The study assessed several factors to determine what risk factors 
were associated with an increase in VAP which included endotracheal tube (ETT) remaining throughout duration of treatment, PRISM score, gram-negative organism present in aspirate, combination antibiotics, additional antibiotic therapy for more than 48 hours within 10 days of VAT diagnosis, and admission to the neonatal intensive care unit. ${ }^{7}$ The only statistically significant prognostic indicator for developing VAP was ETT remaining throughout duration of treatment (adjusted HR 4.16 (1.39-12.45), p < 0.01). ${ }^{7}$ This study, similarly, excluded patients with pre-existing tracheostomies.

In the majority of studies done in patients with VAT, patients with pre-existing tracheostomies have been excluded. These patients are at higher risk of polymicrobial colonization, tracheitis, and pneumonia. ${ }^{8}$ We aimed to describe pediatric patients with pre-existing tracheostomies and determine if there was an increased risk of VAP when treating for 7 days or less, compared to 8 days or more.

\section{Materials and Methods}

A retrospective study was conducted at the Children's Hospital of Georgia (CHOG). The Children's Hospital of Georgia is a 154 bed academic children's hospital serving Georgia and surrounding states. Patients $<18$ years old with pre-existing tracheostomies admitted to the CHOG between January 1, 2007 to February 21, 2021 for initial treatment of VAT were included. Patients were excluded for radiologic evidence of pneumonia within 48 hours prior to VAT treatment, concomitant antibiotics for documented infection other than VAT, or admission to the NICU without discharge home. This study was approved by the Augusta University Medical Center and Children's Hospital of Georgia's Institutional Review Board.

Medical records were retrieved based on ICD-10 codes in order to capture the pediatric patients with preexisting tracheostomies. These ICD-10 codes included tracheostomy dependent (z93.0), ventilator dependent (z99.11), respirator dependent (z99.1), presence of artificial or mechanical lung (z97.8), and complications of tracheostomy (j95.00). From these records, data was gathered including patient demographics, microbiologic data, culture results, antibiotic utilization, and treatment course.

The short duration group received antibiotics for 7 days or less. The long duration group received antibiotics for 8 days or more. The primary outcome was incidence of VAP within 10 days of beginning antibiotics for VAT. In order to get a better understanding of VAT in patients with pre-existing tracheostomies, secondary outcomes included empiric antimicrobial treatment, directed antimicrobial treatment, causative tracheostomy cultures, and previous tracheostomy cultures within two years.

\section{Statistical Analysis}

All statistical analysis was performed using SAS 9.4 and statistical significance was assessed using an alpha level of 0.05. Descriptive statistics by antibiotic duration ([?]7d vs [?]8d) were determined including frequencies and percentages for categorical variables and means and standard deviations for continuous variables. Fisher's Exact tests (due to the small sample size in the [?] 7d and the violation of the assumption for the chi-square test) or t-tests were used to examine differences between antibiotic duration groups. ANOVA analyses were performed for continuous variables across the sample. To examine differences in past cultures and antibiotics disregarding antibiotic duration (to assess antimicrobial stewardship of the entire population), chi-square, or Fisher's Exact if assumptions were violated, and t-tests tests were performed.

\section{Results}

There were one hundred and twenty-three patients screened. Each of the medical records were reviewed to determine if the patient met all inclusion criteria. Thirty-nine patients were included. Eighty-four were excluded, as described in Figure 1. The overall demographics are described in Table 1. Nine patients received 7 days or less of antibiotics. Thirty patients received 8 days or more. There were no significant differences in demographics between the patients receiving antibiotics for 7 days or less, compared to 8 days or more. Across all patients screened, forty-six had a ventilator-associated tracheitis, while thirty-one were excluded for a lack of tracheitis despite having a pre-existing tracheostomy. Across all patients evaluated with a preexisting tracheostomy, there was a $60 \%$ incidence of ventilator associated tracheitis $(46 / 77)$. In the patients experiencing a tracheitis, one patient $(2.5 \%)$ developed VAP, occurring in the group receiving antibiotics 
for 8 days or more. Due to the small rate of occurrence of the primary outcome, no difference could be determined.

The causative bacterial microorganisms of VAT are described in Table 2. The most common organisms were Pseudomonas aeruginosa, Klebsiella pneumoniae, and methicillin-sensitivestaphylococcus aureus (MSSA). Tracheostomy cultures in the previous years are described in Table 3. The most common organisms in the past culture were Pseudomonas aeruginosa, Klebsiella pneumonia, MSSA, and Serratia marcescens . There were no differences in organisms between the short and long duration groups.

The most common empiric antibiotics were cefepime $(n=14)$, ceftriaxone $(n=11)$, and vancomycin $(n=6)$. Chi-square tests were used to examine associations between causative methicillin resistantstaphylococcus aureus (MRSA) in relation to previous tracheostomy culture growing MRSA, empiric vancomycin usage, and positivePseudomonas cultures. The only statistically significant relationship was previous tracheostomy culture growing MRSA ( 1 of 34 with no causative MRSA vs 3 of 5 with causative MRSA, p =0.0042).

Potential demographic risk factors for causative Pseudomonas aeruginosa tracheitis were examined (Table 4). Duration of tracheostomy and age greater than one year of age were associated with $P$. aeruginosa. There was an association between previous $P$. aeruginosa culture and $P$. aeruginosa tracheitis $(\mathrm{p}=0.003) . P$. aeruginosa coverage was defined as an antimicrobial agent that has coverage for $P$. aeruginosa, including piperacillin/tazobactam, cefepime, tobramycin, and ciprofloxacin.. Overall, $65 \%$ (15 of 23 ) of patients with a $P$. aeruginosa were treated with empiric $P$. aeruginosa coverage and $35 \%$ (8 of 23 ) were not covered. As shown in Table 5, there was also an association between previous $P$. aeruginosa culture and empiric $P$. aeruginosa coverage, with $76 \%$ (16 of 21) of patients with previous $P$. aeruginosa cultures receiving empiric coverage, compared to $28 \%$ ( 5 of 18 ) who did not previously grow $P$. aeruginosa

\section{Discussion}

Thirty-nine patients were included in this study and only one developed a ventilator associated pneumonia. This collected data describes patients with pre-existing tracheostomies, in terms of antimicrobial agent choices and microbiome. Additionally, this data can aide in determining how initial presentations with VAT are treated in pediatric patients with pre-exiting tracheostomies.

Previous studies show that in intubated pediatric patients, the incidence of VAT was $7.3 \%{ }^{7}$ Subsequently, VAP was seen in $17 \%$ of pediatric patients following a VAT. ${ }^{7}$ This risk was increased in patients who had an endotracheal tube remaining for the course of therapy. We hypothesized that patients with pre-existing tracheostomies would be at a similarly increased risk. In our patient population, $60 \%(46 / 71)$ of pediatric patients with pre-existing tracheostomies presented with a VAT. However, of our 39 patients, only one developed a VAP. This incidence, $2.5 \%$, is significantly less than originally expected. This suggests that while patients with pre-existing tracheostomies have an increased risk of ventilator associated tracheitis, they may not have an increased risk of ventilator associated pneumonia.

In the population of 39 patients, there were only 5 tracheitis cases with MRSA as a causative organism (13\%). Between June 2017 and February 2021, there were 0 tracheitis cases with MRSA as a causative organism. This could indicate that MRSA colonization is becoming less frequent in pediatric patients at the Children's Hospital of Georgia with pre-existing tracheostomies or this patient population had not yet been exposed to MRSA. Of our total population, thirty-one patients had a pre-existing tracheostomy but no tracheitis, which could indicate that patients with tracheostomies outside of the hospital are less likely to present to the hospital with a tracheitis, and thus less likely to be exposed to multi-drug resistant organisms such as MRSA. The only statistically significant risk factor for MRSA tracheitis was previous MRSA culture, which suggests that empiric MRSA coverage should be added in patients with a previous MRSA culture. In this patient population, only 2 of 5 patients (40\%) were empirically treated with vancomycin, whereas 4 of 5 patients $(80 \%)$ had a previous MRSA culture, suggesting that empiric treatment was not guided by previous cultures.

Pseudomonas aeruginosa was the most common causative organism of all tracheitis, with $59 \%$ of patients 
having a positive $P$. aeruginosa culture. $P$. aeruginosa was more common in children younger than 1 year old and significantly associated with tracheostomy duration, with a longer duration of tracheostomy being associated with a greater risk of $P$. aeruginosa. This could mean that as patients are exposed to the health system and more organisms, they are at increased risk of $P$. aeruginosa infection. $P$. aeruginosatracheitis was associated with a previous $P$. aeruginosa culture $(\mathrm{p}=0.0003)$. However, 5 of 21 patients $(24 \%)$ with a previous tracheostomy culture growing $P$. aeruginosa were not empirically covered for $P$. aeruginosa. In patients who had a $P$. aeruginosa tracheitis, 8 of $23(35 \%)$ were not given coverage for $P$. aeruginosa. Due to the high percentage of patients growing $P$. aeruginosa, $P$. aeruginosa should be considered in patients with pre-existing tracheostomies presenting with upper respiratory infections. On a larger scale, institutions can re-assess their trends in ventilator-associated tracheitis causative organisms to re-assess treatment pathways and risk factors in pediatric patients with pre-existing tracheostomies in order to appropriately empirically treat them.

This data indicates that treatment for tracheitis should be guided by previous cultures, given both MRSA and $P$. aeruginosa infections were statistically significantly associated with previous cultures for MRSA and $P$. aeruginosa, respectively. At our institution, due to the low incidence of MRSA overall, and especially in recent years, empiric coverage for MRSA is not necessary in all patients. WithP. aeruginosa being so prevalent and 8 of $23(35 \%)$ of patients requiring $P$. aeruginosa coverage without receiving it, initial empiric therapy should include $P$. aeruginosa coverage.

This study had several limitations. No difference could be determined for VAP due to the low incidence in both antibiotic duration groups. Additionally, the retrospective nature over a very long range of time could be limited by practice changes over time, with duration of treatment getting gradually shorter as time progressed. Additionally, in order to prevent confounding that could happen from including the same patient over multiple admissions, only the initial inpatient treatment for VAT was included. Additional studies are needed to determine the optimal treatment duration for VAT in pediatric patients with preexisting tracheostomies and how the organisms and treatment may differ with repeat admissions. There were several strengths to this study. Patients with pre-existing tracheostomies are frequently excluded from adult and pediatric trials. This study adds information on incidence of ventilator associated tracheitis, incidence of ventilator associated pneumonia, microbial biome, risk factors forPseudomonas aeruginosa, and antimicrobial utilization in this specific subset of patients to the current literature. Additionally, the ability to compare treatment with previous and causative cultures allows for potential modification of treatment pathways in patients presenting with an initial VAT.

Ventilator associated pneumonia is a common cause of healthcare costs and morbidity in pediatric patients. Patients with pre-existing tracheostomies represent a gap in current literature. Although there are numerous studies assessing treatment of VAP and VAT across adult and pediatric studies, these studies frequently exclude patients with pre-existing tracheostomies. This study did not find a difference in VAP in pediatric patients with pre-existing tracheostomies. Across the study population, the incidence of VAT was significantly higher than what has been shown in pediatric patients without tracheostomies and the incidence of VAP was lower than what was expected based on literature. The analysis of previous and causative culture results strengthens the benefit of empirically treating based on previous culture results. Additionally, the high percentage of patients presenting with a $P$. aeruginosa tracheitis without appropriate coverage indicates that empiric therapy for $P$. aeruginosa should be considered for the initial hospitalization with VAT. Future studies are needed to determine differences across repeat hospitalizations for VAT and the optimal duration of treatment for VAT to prevent a VAP.

Acknowledgements: The authors have no conflicts of interest to disclose.

\section{References:}

1. Agrafiotis, M., I.I. Siempos, and M.E. Falagas, Frequency, prevention, outcome and treatment of ventilator-associated tracheobronchitis: Systematic review and meta-analysis. Respiratory Medicine, 2010. 104(3): p. 325-336. 
2. Cheikh, M.R.E., et al., Microbiology of Tracheal Secretions: What to Expect with Children and Adolescents with Tracheostomies. International Archives of Otohinolaryngology, 2017. 22: p. 50-54.

3. Donald and Karin, Ventilator-Associated Tracheobronchitis and Pneumonia: Thinking Outside the Box. Clinical Infectious Diseases, 2010. 51(S1): p. S59-S66.

4. Koenig, S.M. and J.D. Truwit, Ventilator-Associated Pneumonia: Diagnosis, Treatment, and Prevention. Clinical Microbiology Reviews, 2006. 19(4): p. 637-657.

5. Nseir, S., et al., Antimicrobial treatment for ventilator-associated tracheobronchitis: a randomized controlled multicenter study. Critical Care, 2008. 12(3): p. R62.

6. Nseir, S., et al., Impact of appropriate antimicrobial treatment on transition from ventilator-associated tracheobronchitis to ventilator-associated pneumonia. Critical Care, 2014. 18(3): p. R129.

7. Tamma, P.D., et al., Ventilator-Associated Tracheitis in Children: Does Antibiotic Duration Matter? Clinical Infectious Diseases, 2011. 52(11): p. 1324-1331.

\section{Hosted file}

Final Tables.docx available at https://authorea.com/users/421468/articles/527492-evaluationof-ventilator-associated-tracheitis-in-children-with-pre-existing-tracheostomies-

organisms-and-empiric-coverage 